\title{
O Grupo costatus de Ommatius Wiedemann (Diptera, Asilidae) no Brasil: Novos Registros e Distribuição de Ommatius costatus Rondani e Ommatius orenoquensis Bigot
}

\begin{abstract}
Rodrigo Vieira
Instituto Nacional de Pesquisas da Amazônia, e-mail: rodrigoo8vieira@gmail.com.

\section{EntomoBrasilis 8 (1): 58-64 (2015)}

Registrado no ZooBank: urn:lsid:zoobank.org:pub:80o66B5D-F914-4B85-B297-527967899EF5

Resumo. Os limites geográficos de quatro espécies de Ommatiinae são estendidos: Ommatius complanatus Scarbrough, 1993; Ommatius spatulatus Curran, 1928; Ommatius spinosus Scarbrough, 1993; Ommatius uncatus Scarbrough, 1993. Além disso, um espécime adicional de Ommatius didymus Scarbrough, 1993 é examinado e suas variações morfológicas são anotadas. São fornecidos ainda registros geográficos de Ommatius costatus Rondani e Ommatius orenoquensis Bigot para o Brasil.
\end{abstract}

Palavras-chaves: Distribuição; Ommatiinae; Taxonomia.

\section{The costatus Group of Ommatius Wiedemann (Diptera, Asilidae) in Brazil: New Records and Distribution of Ommatius costatus Rondani and Ommatius orenoquensis Bigot}

Abstract. The geographic ranges of four species of Ommatiinae are extended: Ommatius complanatus Scarbrough, 1993; Ommatius spatulatus Curran, 1928; Ommatius spinosus Scarbrough, 1993; Ommatius uncatus Scarbrough, 1993. Furthermore, an additional specimen of Ommatius didymus Scarbrough, 1993 is examined and their morphological variations are noted. Geographical records of Ommatius costatus Rondani, 1850 and Ommatius orenoquensis Bigot, 1876 are listed from Brazil.

Keywords: Distribution; Ommatiinae; Taxonomy.

( mmatiinae Hardy, 1927 é uma das 14 subfamílias de Asilidae (Dıкоw 2009) e atualmente é constituída por 11 gêneros: Afroestricus Scarbrough, 2005, Cophinopoda Hull, 1958, Emphysomera Schiner, 1866, Merodontina Enderlein, 1914, Michotamia Macquart, 1838, Ommatius Wiedemann, 1821, Ommatomyia Scarbrough \& Tomasovic, 2010, Pseudomerondontina Joseph \& Parui, 1976, Pygommatius Scarbrough \& Marascia, 2005, Stenommatius Matsumura, 1916, Thallosia Oldroyd, 1970 (Scarbrough \& Tomasovic 2010). Afroestricus, Pygommatius e Thallosia são registrados para a região Afrotropical, Merodontina, Ommatomyia, Pseudomerondontina e Stenommatius para a região Oriental, Michotamia e Emphysomera para as regiões Afrotropical, Australasia e Oriental, Cophinopoda para as regiões Afrotropical, Australasia, Oriental e Paleártica, e por fim Ommatius possui uma ampla distribuição, ocorrendo em quase todas as regiões biogeográficas, exceto o Chile (Scarbrough \& Tomasovic 2010).

Ommatius é bem diversificado e necessita de uma revisão. Além disso, é reconhecido apenas pelo estilo da antena plumosa (autapomorfia de Ommatiinae) (SCARBROUGH \& MARASCIA 2003; SCARBrough 2005; Dikow 2009). Este gênero possui 317 espécies válidas para o mundo, 117 para a região Neotropical e 24 para o Brasil (VIEIRA et al. 2004, 2005, 2010, 2011).

$\mathrm{Na}$ classificação de Ommatius são reconhecidos 10 grupos, oito para as Américas e apenas três para o Brasil: costatus, holosericeus e normus (SCARBROUGH 1990, 1993, 2000, 2002, 2008; Scarbrough \& Constantino 2005; Scarbrough \& PerezGELABERT 2006)
Neste trabalho é descrita uma variação de Ommatius didymus Scarbrough, são fornecidos novos registros geográficos para o Brasil de quatro espécies de Ommatius do grupo costatus, assim como se detalha, com base na informação das etiquetas afixadas aos espécimes, as localidades de coleta de duas espécies, Ommatius costatus Rondani e Ommatius orenoquensis Bigot. Estas espécies por possuírem uma ampla distribuição na região Neotropical, são citadas em trabalhos anteriores apenas como ocorrendo no "Brasil".

\section{MATERIAL E MÉTODOS}

Foram examinados exemplares das seguintes instituições: Coleção de Invertebrados do Instituto Nacional de Pesquisas Amazônia, Manaus, Brasil (INPA); Coleção Entomológica Professor Johann Becker do Museu de Zoologia da Universidade Estadual de Feira de Santana, Feira de Santana, Brasil (MZUEFS); Museu de Zoologia da Universidade de São Paulo, São Paulo, Brasil (MZUSP); Museu Nacional da Universidade Federal do Rio de Janeiro, Rio de Janeiro, Brasil (MNRJ); Coleção Entomológica Padre Jesus Santiago Moure do Departamento de Zoologia da Universidade Federal do Paraná, Curitiba, Brasil (DZUP); Museu Paraense Emílio Goeldi, Belém, Brasil (MPEG); Universidade de Santa Cruz do Sul, Santa Cruz do Sul, Brasil (UNISC); The Field Museum of Natural History, Chicago, EUA (CFMNH); The Natural History Museum, Londres, Reino Unido (NHM).

Agências de Financiamento: PPBio, CNPq e a FAPEAM EDITAL N. 022/2013 - FIXAM/AM No Processo: 062.00745/2014 
T A técnica de VieIRA (2012) foi utilizada para examinar a terminália. Após estudo e ilustração, a terminália foi acondicionada em microtubo contendo glicerina e este foi afixado ao alfinete do espécime correspondente. A terminologia adotada segue CumMING \& WoOD (2009).

Para a relação dos dados das etiquetas dos espécimes, os nomes dos países foram colocados em letras maiúsculas, os estados em negrito, os meses de coletas em algarismo romano minúsculo e o número de espécimes, sexo e a instituição à qual pertencem em negrito e entre parênteses. Os dados de cada etiqueta dos espécimes foram separados por uma barra / e as informações úteis adicionais, não encontradas nestas, foram colocadas entre colchetes []. Além disso, correções de dados das etiquetas foram informadas através de chaves \{\} . Devido ao grande número de espécimes encontrados, os dados das etiquetas de $O$. costatus e O. orenoquensis foram reduzidas apenas às localidades.

Os registros geográficos marcados com asterisco * correspondem aos dados de etiquetas que não puderam ser associadas com municípios ou localidades, constando somente os dados originais das mesmas.

\section{RESULTADOS E DISCUSSÃO}

\section{Ommatius complanatus Scarbrough, 1993}

Ommatius complanatus Scarbrough, 1993: 736, figs 23-33; 2007: 471; Vieira, Castro \& Bravo, 2005: 20 (chave); Papavero, 2009: 48 (catálogo).

Comentários. O. complanatus é registrada pela primeira vez para o estado do Paraná. O espécime encontrado tem uma variação quando comparado ao holótipo. O fêmur mediano possui duas cerdas castanhas finas na região ântero-ventral e três cerdas amareladas no tarsômero basal anterior, enquanto que, no holótipo, o fêmur mediano possui uma cerda castanha fina na região ântero-ventral e duas cerdas amareladas no tarsômero basal anterior.

Distribuição. BRASIL: São Paulo: Cidade Jardim*, Ferraz de Vasconcelos, Salesópolis. Rio de Janeiro: Rio de Janeiro. Paraná: Telêmaco Borba (novo registro).

Material examinado. Holótipo: [BRASIL] Est.[ação] Biol. Boracéia, Salesópolis, SP [São Paulo], 850m, Rabello Col.[etor], 30.i.[19]68 / Holotype Ommatius complanatus Scarbrough (10 MZUSP); Parátipo: [BRASIL], Alto da Boa Vista, Tijuca (DF) \{antiga sede do Distrito Federal do Brasil, [Rio de Janeiro]\}, iii.1950, C.A.C. Seabra col.[etor] / Paratype Ommatius complanatus Scarbrough (1 $\widehat{\jmath}, 1+$ MZUSP).

Material adicional. DZUP 181641 / Telêmaco Borba, PR [Paraná], Res. Samuel Klabin, BRASIL, 29.xi.1986, Lev. Ent. profaupar, lâmpada / Ommatius complanatus Det. R. Vieira, 2007 ( 1 ^ DZUP).

\section{Ommatius costatus Rondani, 1850 (Figura 1)}

Ommatius costatus Rondani, 1850: 188; Osten Sacken, 1891: 421; Kertész, 1909: 307 (catálogo); Hull, 1962: 435; Martin \& Papavero, 1970: 59 (catálogo); Scarbrough, 1993: 738, figs 3446; 2007: 471; Vieira, Castro \& Bravo, 2005: 21 (chave); Vieira, Castro, Almeida, Alvim \& Bravo, 2006: 247, figs 18; Papavero, 2009: 48 (catálogo).

Ommatius barbiellinii Curran, 1934: 18; Bromley, 1946: 112; Hull, 1962: 435; Martin \& Papavero, 1970: 59, (catálogo); Papavero, 2009: 48 (catálogo).

Comentários. $O$. costatus (Figura 1) é amplamente distribuída na região Neotropical. ScARBRough (1993), redescreveu $O$. costatus baseado em vários espécimes de diversas coleções, no entanto, forneceu apenas a distribuição geográfica da espécie, sem fornecer os registros geográficos específicos. Com base no material examinado neste trabalho, é possível notar que $O$. costatus possui uma ampla distribuição no Brasil, ocorrendo do Norte ao Sul do país (Figura 2).

Distribuição. TRINIDAD, América do Sul exceto CHILE e URUGUAI. BRASIL: Amazonas: Manaus. Pará: Tucuruí (Bagagem), Tucuruí (Ilha Chorona). Mato Grosso: Utiariti (Rio Papagaio), Cáceres. Goiás: Goiânia, Corumbá de Goiás, Jataí. Minas Gerais: Aimorés, Araçaí, Arceburgo, Governador Valadares, Macaúbas, Pouso Alegre, Santa Rita de Caldas. Mato Grosso do Sul: Maracajú, Salobra [Miranda]. Espírito Santo: Santa Teresa. São Paulo: Andes, Araçatuba (Córrego Azul), Araçatuba (Rio Jacaretinga), Batatais, Barueri, Caieiras, Cajuru, Cássia dos Coqueiros, Caraguatatuba, Castilho (margem esquerda do Rio Paraná), Cidade Jardim*, Cotia, Guarujá, Guatapará, Ilha da Vitória, Ilha de Búzios, Itaporanga, Juquiá, Mogi das Cruzes, Nova Granada, Osasco, Prainha Branca (Ilha de Santo Amaro), Ribeirão Preto, Rio Claro, São Paulo, São Sebastião (Ilha Bela), São Vicente (Praia das Vacas), Serra de Santos (Km 48), Severinia, Tabatinga, Vera Cruz, Ubatuba, Várzea*. Rio de Janeiro: Angra dos Reis (Japulyba), Angra dos Reis, Serra de Angra, Tinguá [Nova Iguaçu], Itatiaia, Magé, Muriqui [Niterói], Nova Friburgo (Mury), Rio de Janeiro, Santa Maria Madalena (Santo Antônio do Imbé). Paraná: Guaratuba, Rio Pacaraí. Santa Catarina: Brusque, Florianópolis, Itajaí, Joinville, Nova Teutônia [Seara], Rio das Antas. Rio Grande do Sul: Pelotas.

Material examinado. Amazonas: Manaus $(1 \hat{\jmath}, 1$ 우 MZUSP, 1 우 INPA). Pará: Tucuruí (Bagagem) (1우 INPA), Tucuruí (Ilha Chorona) (1 $\delta^{\wedge}$ INPA). Mato Grosso: Utiariti (Rio Papagaio) (1우 MZUSP), Cáceres (1ㅇํㅁZUP). Goiás: Campinas \{Goiânia\} (2ㅇ MZUSP), Corumbá [de Goiás] (5 $\hat{\circ}, 12$ 우 MZUSP), Jataí (2ㅇ MZUSP). Minas Gerais: Aimorés (1 $\sigma^{\Uparrow}$ MZUSP), Araçaí (1ㅇ MZUSP), Arceburgo ( 5 을 MZUSP), Governador Valadares (2우 MZUSP), Macaúba [Mosteiro de Macaúbas] (1 $q$ MZUSP), Pouso Alegre (6 ${ }^{\lambda}, 43 \circ$ MZUSP), Santa Rita de Caldas (2 9 MZUSP). Mato Grosso do Sul: Maracajú ( $1 \hat{\jmath}, 6 \circ$ MZUSP), Salobra [Miranda] (1우 MZUSP). Espírito Santo: Santa Teresa (5우 MZUSP). São Paulo: Andes (1오 MZUSP), Araçatuba (Córrego Azul) (1 $\hat{\jmath}, 3$ 우 MZUSP), Araçatuba (Rio Jacaretinga) (2 9 MZUSP), Batatais ( $1 \overbrace{}^{\lambda} \mathrm{DZUP})$, Barueri $\left(6{ }^{\lambda}, 13 ㅇ\right.$ MZUSP), Caieiras (1우 MZUSP), Cajuru, Cássia dos Coqueiros (3ð MZUSP), Caraguatatuba (3우 MZUSP), Castilho (margem esquerda do Rio Paraná) (2 $\widehat{\jmath}, 2$ + MZUSP), Cidade Jardim* (2ㅇ MZUSP), Cotia (1ㅇ MZUSP), Guarujá (4ㅇ MZUSP), Guatapará (2 $\hat{\jmath}, 2$ ㅇ MZUSP), Ilha da Vitória (1우 MZUSP), Ilha de Búzios (1 $\jmath^{\lambda}, 7$ 우 MZUSP), Itaporanga ( $3 \hat{\jmath}, 6+$ MZUSP), Juquiá (1우 MZUSP), Mogi das Cruzes $(1 \hat{\jmath}, 1+$ MZUSP), Nova Granada (1우 MZUSP), Osasco (10ㅅ, 4우 MZUSP),

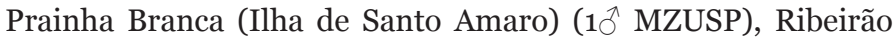

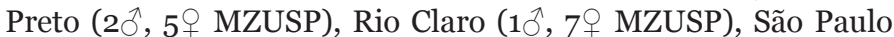
(2犬̂, 1 ㅇ MZUSP), São Sebastião (Ilha Bela) (1 9 MZUSP), São Vicente (Praia das Vacas) (1우 MZUSP), Serra de Santos (Km 48)

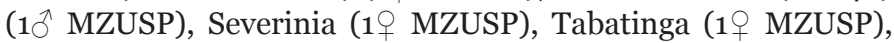
Vera Cruz (2 9 MZUSP), Ypiranga* [Ipiranga, São Paulo] (1 $\partial^{\lambda}, 3$ 우

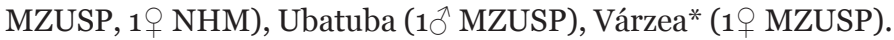
Rio de Janeiro: Angra dos Reis (Japuíba) (1 $\hat{\jmath}, 5 \circ$ MZUSP),

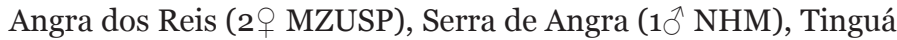

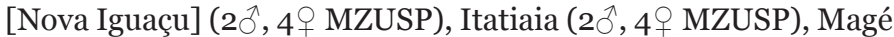

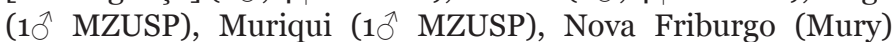
$(3 \hat{\jmath}, 4 \uparrow$ MZUSP), Rio de Janeiro (10, $1 \uparrow$ MZUSP), Santa Maria Madalena (Santo Antônio do Imbé) (10̄ MZUSP). Paraná: Guaratuba ( $1 \hat{\sigma}$ MZUSP), Rio Pacaraí (3 $\hat{\sigma}$ MZUSP). Santa Catarina: Brusque ( $2 q$ MZUSP, $2 q$ DZUP), Florianópolis $(1 \hat{\jmath}$,

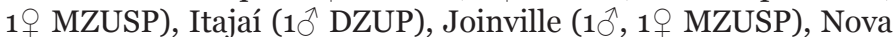

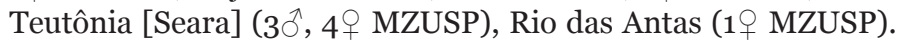
Rio Grande do Sul: Pelotas $(1 \overbrace{}^{\lambda}, 1+$ MZUSP).

\section{Ommatius didymus Scarbrough, 1993 (Figura 3)}

Ommatius didymus Scarbrough, 1993: 741, figs 58-65; 2007: 471; Vieira, Castro \& 
Bravo, 2005: 21 (chave). Papavero, 2009: 49 (catálogo).

Comentários: A descrição original de $O$. didymus foi baseada apenas no holótipo macho (Figura 3) (SCARbrough 1993). Neste trabalho foi encontrado outro exemplar macho de $O$. didymus também proveniente do estado do Amazonas que contém as seguintes variações: corpo com $13 \mathrm{~mm}$ e asa com 10,5 mm; corpo enegrecido; místax com seis cerdas pretas e longas e duas cerdas pretas, curtas e finas; duas cerdas pós-alares (uma longa e uma curta, medindo cerca de 1/3 o comprimento da outra); três pares de cerdas dorsocentrais; pleura com tomento cinza; veia costal da asa dilatada na porção mediana; fêmur mediano com uma cerda ântero-ventral; fêmur posterior com 2/3 basal amarelado e 1/3 apical preto, região ântero-ventral sem uma cerda basal amarelada, com somente seis cerdas pretas ântero-ventrais. No holótipo, o corpo com $12.1 \mathrm{~mm}$ e asa com $10 \mathrm{~mm}$; o corpo é castanho-avermelhado; o místax possui apenas seis cerdas pretas e longas; uma cerda pós-alar, quatro pares de cerdas dorsocentrais; pleura castanha, com tomento cinza e esparso; veia costal da asa levemente dilatada na porção mediana; fêmur mediano com duas cerdas ântero-ventrais; fêmur posterior com a metade basal amarelada e metade apical marrom, região ântero-ventral com sete cerdas ântero-ventrais, sendo uma basal amarelada e as demais pretas.

\section{Distribuição. BRASIL: Amazonas.}

Material examinado: Holótipo: 2876/ BRASIL, AM[azonas], Manaus, Est.[rada] AM 1. Km 134, 10.vii.1968 Col[etor]? / Holotype Ommatius didymus Scarbrough (10 INPA).

Material adicional. BRASIL, Amazonas, AM-010, Km 31, Embrapa, 19.ii.1992, L. P.

Albuquerque, J. Binda [Coletor] / Arm.[adilha]Shannon, Capoeira, Isc.[a] Fruta 19.ii.1992 / Ommatius didymus Det. R.

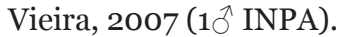

\section{Ommatius orenoquensis Bigot, 1876 (Figura 4)}

Bigot, 1876: 1xxxv; Osten Sacken, 1891: 421; Kertész, 1909: 310 (catálogo); Hull, 1962: 435; Martin \& Papavero, 1970, 35b: 59 (catálogo); Scarbrough, 1993: 746, figs 88-98; 2007: 471; Vieira, Castro \& Bravo, 2005: 20 (chave); Vieira, Castro, Almeida, Alvim \& Bravo, 2006: 251; Papavero, 2009: 51 (catálogo); Cezar \& Lamas, 2010: 28 (descrição de imaturos)

Ommatius infractus Scarbrough, 1985: 643, figs 2-6, 1993: 746; Scarbrough, 1993: 746; Vieira, Castro \& Bravo, 2005: 20; Vieira Castro, Almeida, Alvim \& Bravo, 2006: 252; Papavero, 2009: 51 (catálogo).

Comentários. Espécimes de O. orenoquensis (Figura 4), juntamente com espécimes de $O$. costatus e $O$. pulcher (Engel, 1885) são encontrados em grande quantidade nas coleções entomológicas brasileiras. Scarbrough (1993) redescreveu $O$. orenoquensis e fez comentários da sua ampla distribuição, no entanto não forneceu os registros geográficos da espécie. $O$. orenoquensis é amplamente distribuída na região Neotropical e no Brasil, no qual ocorre desde Roraima até o Rio Grande Sul (Figura 5).

Distribuição. COSTA RICA, PANAMÁ, TRINIDAD, TOBAGO, GUIANA FRANCESA, Sul da ILHA DE SÃO VICENTE, América do Sul exceto CHILE e URUGUAI. BRASIL: Roraima: Rio Uraricoera (Ilha de Maracá, Município de Amajari). Amazonas: Manaus, Itacuraça*, Parque Nacional do Jaú [Nova Airão e Barcelos], ZF-03, Ceplac (Estrada de Itacoatiara), Nova Aripuaranã. Pará: Conceição do Araguaia, Óbidos, Rio Acará, Oriximiná (Boca do Cuminá-Miri), Rodovia Belém-Brasilia Km 90 F Candiru, Vigia, Santarém. Rondônia: V. Rondônia 378 Km $\mathrm{S}$ de Porto Velho. Mato Grosso: $12^{\circ} 50$ 'S $51^{\circ} 45^{\prime} \mathrm{W}$ (Bom Jesus do Araguaia). Bahia: Barreiras, Barrolândia, Cachoeira, Feira de Santana, Senhor do Bonfim, Una, Itaberaba, Itabuna, Ituberá, Lençóis, Mata de São João, Morro do Chapéu, Salvador, Sauípe, Porto Seguro. Minas Gerais: Aimorés, Alpinópolis, Pratapólis. Mato Grosso do Sul: Três Lagoas. Espírito Santo: Baixo Guandu, Conceição da Barra, Santa Teresa, Vila Velha, Vitória. São Paulo: Luiz Antonio, Piracicaba, Ubatuba. Rio de Janeiro: Angra dos Reis, Duque de Caxias, Rio de Janeiro, Tinguá [Nova Iguaçu]. Paraná: Apucarana, Jundiaí do Sul, Guarapuava (Est. Águas Santa Clara). Santa Catarina: Nova Teutônia [Seara]. Rio Grande do Sul: Santa Cruz do Sul, Santa Maria.

Material examinado. Roraima: Rio Uraricoera, Ilha de Maracá [Município de Amajari] (1 $\widehat{O}^{\lambda}$ INPA). Amazonas:

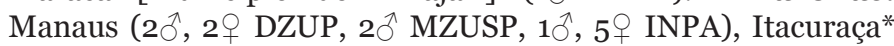

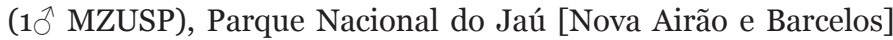

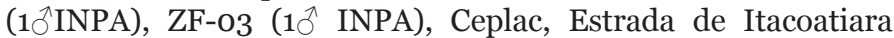
(1우 INPA), Nova Aripuaranã (20̂̉ INPA). Pará: Conceição do Araguaia ( $1 \bigcirc^{\Uparrow}$ INPA), Óbidos (1요 INPA), Rio Acará ( 2 ㅇ MZUSP), Oriximiná (Boca do Cuminá-Miri) (2q MZUSP), Rodovia Belém-

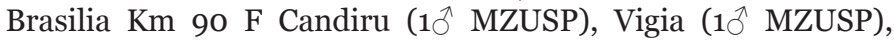
Santarém (1ㅇ MZUSP). Rondônia: V. Rondônia $378 \mathrm{Km}$ S de Porto Velho (1ㅇ MZUSP). Mato Grosso: $12^{\circ} 50^{\prime} S 51^{\circ} 45^{\prime} \mathrm{W}$

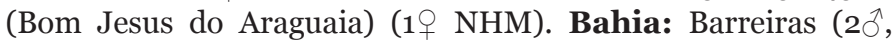

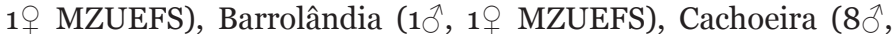
11 ㅇ MZUEFS), Feira de Santana (2 $\hat{0}, 4+$ MZUEFS), Senhor do Bonfim (6 $\hat{\jmath}, 4+$ MZUEFS), Una (1우 MZUEFS), Itaberaba (2우 MZUEFS), Itabuna ( $1 \hat{\jmath}$ MZUEFS), Ituberá (4 $\hat{\jmath}, 17$ 우 MZUEFS), Lençóis ( $5 \hat{\jmath}, 6$ ㅇ MZUEFS), Mata de São João (1 $\hat{\sigma}^{\hat{\gamma}}$ MZUEFS), Morro do Chapéu (5우 MZUEFS); Salvador (10, 6 우 MZUEFS,

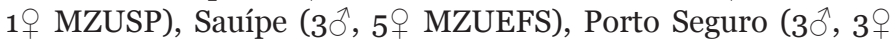

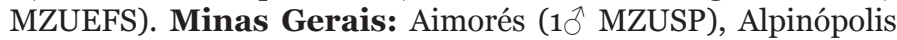

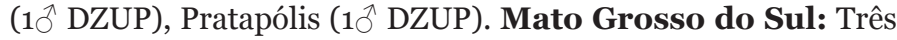
Lagoas (1 $\overbrace{}^{\lambda}$ MZUSP). Espírito Santo: Baixo Guandu (2 $\hat{\jmath}, 3$ 우

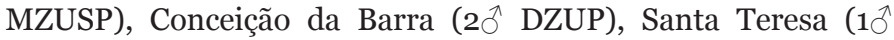
DZUP), Vila Velha (1 $\hat{\jmath}$ MZUEFS), Vitória (2 $\widehat{\jmath}$ MZUEFS). São Paulo: Piracicaba ( $1 \hat{\jmath}$ MZUSP), Ubatuba ( $1 \hat{\jmath}$ MZUSP). Rio de

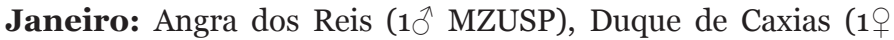

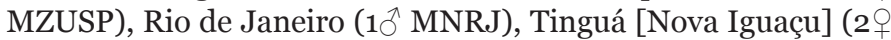
MZUSP). Paraná: Apucarana (1요 INPA), Jundiaí do Sul $(1 \hat{\jmath}, 1$ 우 DZUP), Guarapuava (Est. Águas Santa Clara) (10̄ DZUP). Santa Catarina: Nova Teutônia [Seara] $(12 \hat{\gamma}, 20 \bigcirc$ MZUSP, 2 ㅇ NHM, $5 \hat{\jmath}, 4$ CFMNH, $1 \hat{\jmath}, 1$ 우 DZUP). Rio Grande do Sul: Santa

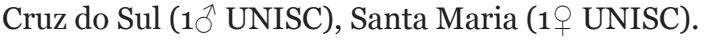

\section{Ommatius spatulatus Curran, 1928}

Curran, 1928: 4; Bromley, 1946: 112 (catálogo); Carrera, 1960a: 167; Hull, 1962: 435; Martin \& Papavero, 1970: 6o (catálogo); Scarbrough 1993: 731; 2007: 470; Vieira, Castro \& Bravo 2005: 19; Papavero, 2009: 52 (catálogo).

Ommatius riali Vieira, Castro \& Bravo, 2005: 21; Papavero, 2009: 51 (catálogo).

Comentários. O. spatulatus é registrada pela primeira vez para os estados de Roraima e Goiás.

Distribuição. BRASIL: Roraima: Pacaraima (novo registro). Amazonas: Vista Alegre [Canutama] (Rio Blanco). Mato Grosso: Diamantino. Bahia: Cachoeira, Mucuri. Goiás: Corumbá de Goiás (novo registro). Minas Gerais: Arceburgo, Belo Horizonte, Brumadinho, Macaúba, Pouso Alegre, Tumiritinga. Mato Grosso do Sul: Maracajú. Espírito Santo: Baixo Guandu, Conceição da Barra, Itaguaçú, Linhares, São Mateus. São Paulo: São Paulo, Barueri, Caraguatatuba, Ribeirão Preto, Ilha de Búzios. Rio de Janeiro: Angra dos Reis, Itatiaia, Rio de Janeiro, Ilha do Governador, Itaguaí, Santa Maria Madalena (Santo Antônio do Imbé), km 47 Estrada Rio de Janeiro-São Paulo. Rio Grande do Sul: Santa Cruz do Sul. ARGENTINA.

Material examinado. Roraima: Pacaraima ( $1 \hat{\circ}$ INPA). Mato Grosso: Diamantino, (1 $\widehat{\jmath}$ DZUP). Bahia: Cachoeira ( $1 \circlearrowleft$ 

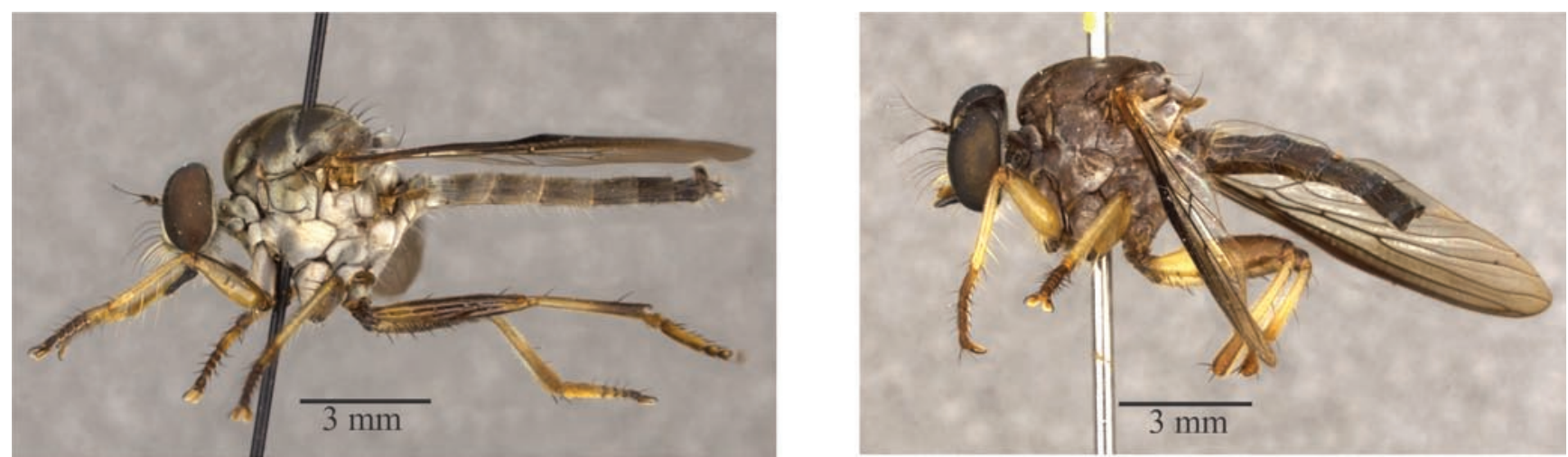

$\mathbf{1}$

3

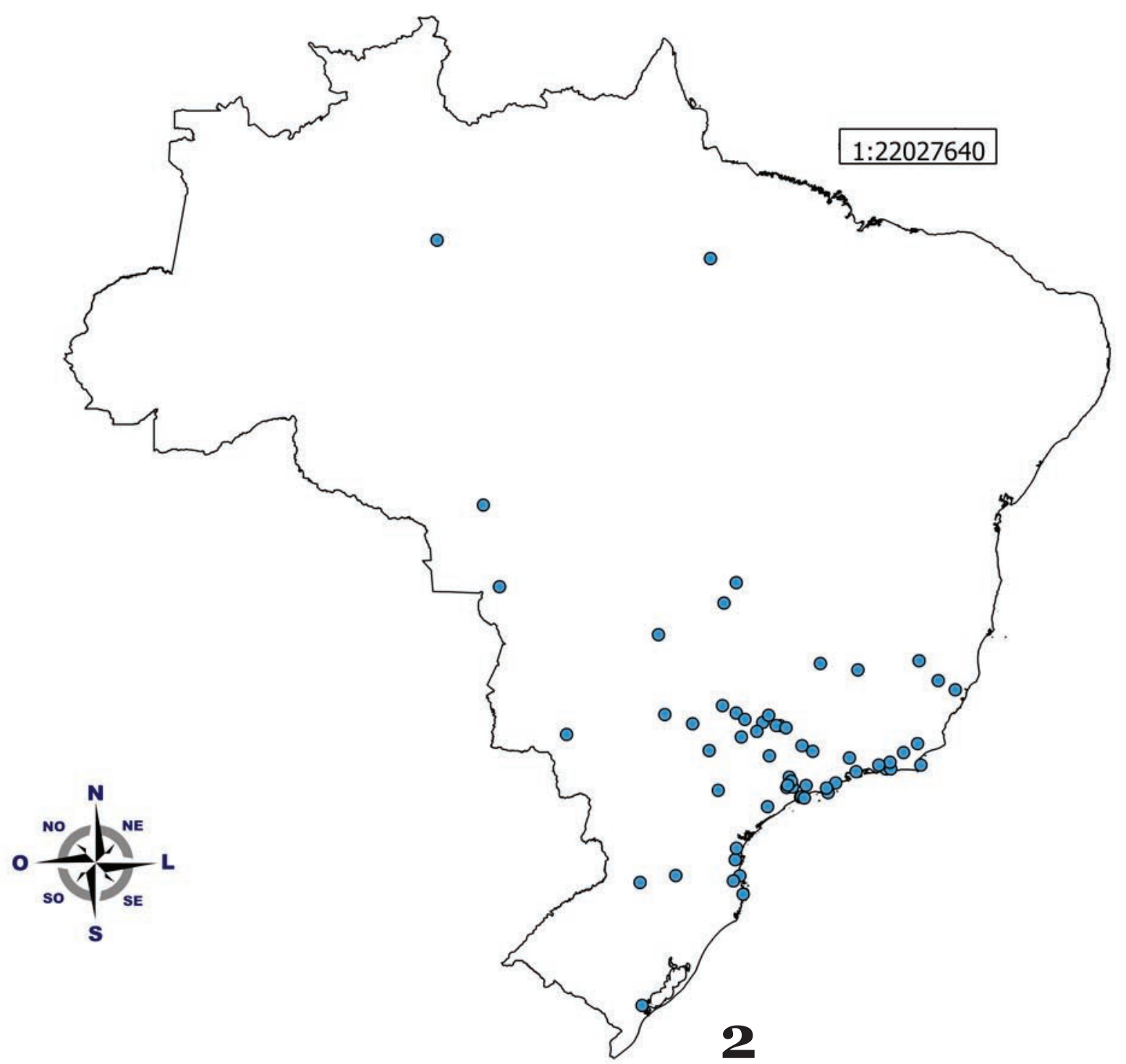

Figuras 1-3. 1. Ommatius costatus Rondani, 1850, hábito do macho em vista lateral; 2. Mapa dos registros geográficos de Ommatius costatus no 5 Brasil; 3. Ommatius didymus Svarbrough, 1993, Hábito do holótipo macho em vista lateral. 

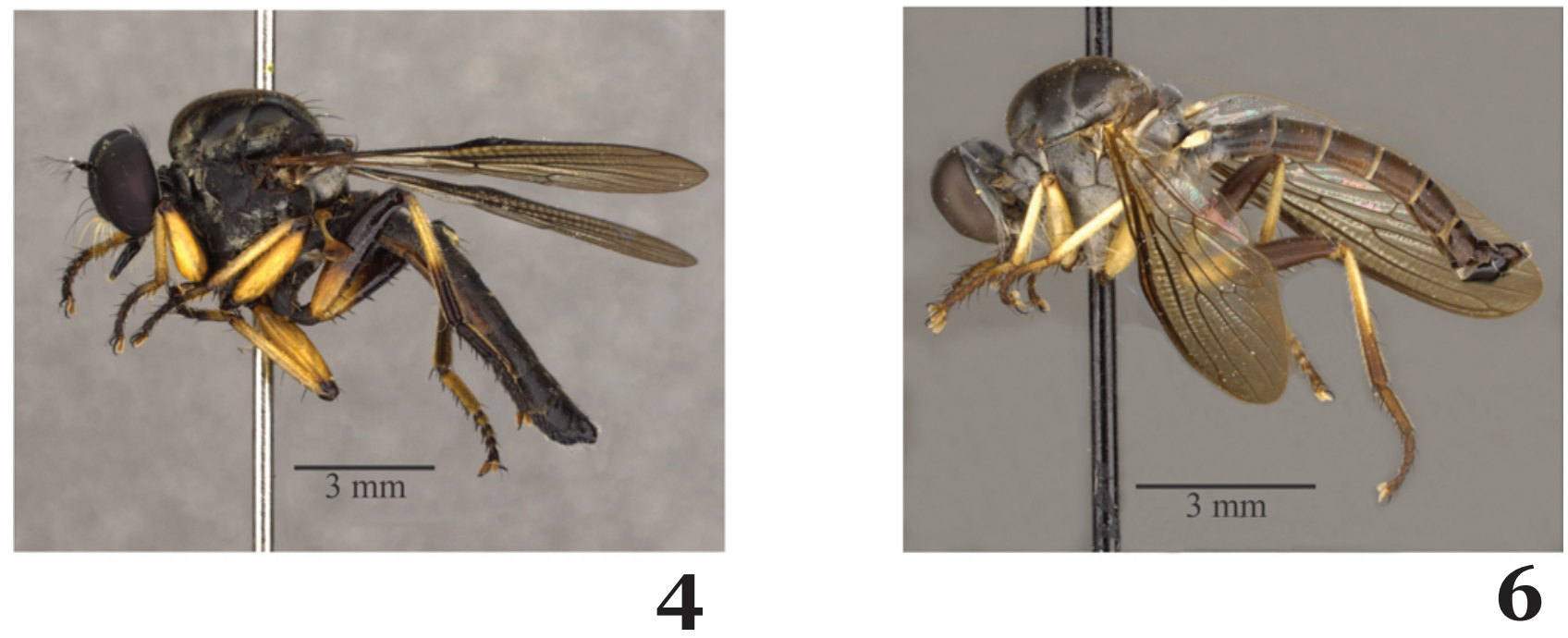

6

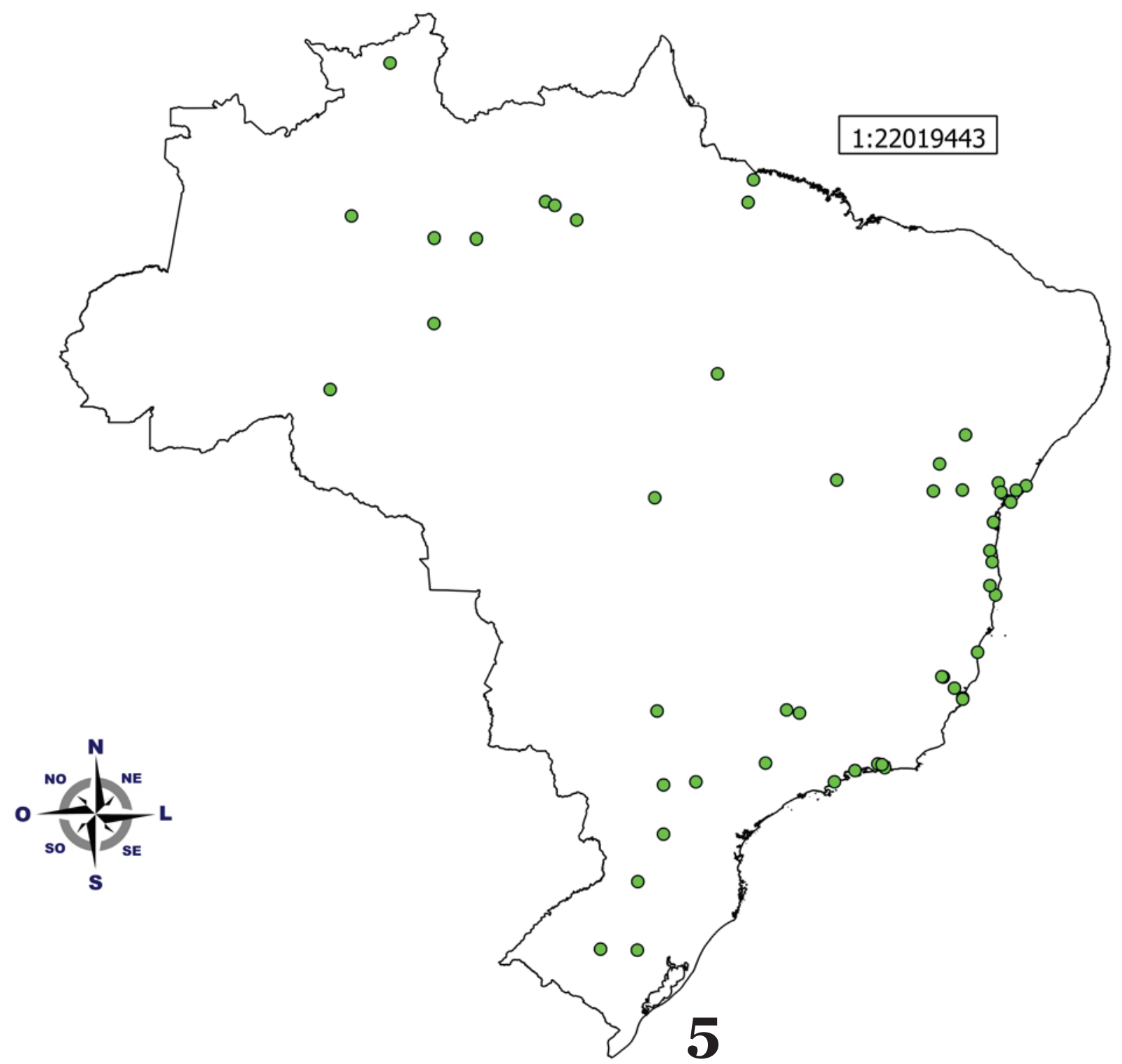

Figuras 4-6. 4. Ommatius orenoquensis Bigot, 1876, hábito do macho em vista lateral; 5. Mapa dos registros geográficos de Ommatius orenoquensis no Brasil; 6. Ommatius spinosus Scarbrough, 1993, hábito do parátipo macho em vista lateral. 
MZUEFS), Mucuri (4우 MZUSP). Goiás: Corumbá [de Goiás] (1 $\Uparrow$ MZUSP). Minas Gerais: Arceburgo (4 MZUSP), Macaúba (3ํ MZUSP), Pouso Alegre (4 $\overbrace{}^{\Uparrow}$ MZUSP), Brumadinho (3ㅇ MZUSP). Mato Grosso do Sul: Maracajú ( $3 \hat{\jmath}, 3$ ㅇ MZUSP). Espírito Santo: São Mateus (1 $\overbrace{}^{\lambda}, 1+$ DZUP), Baixo Guandu

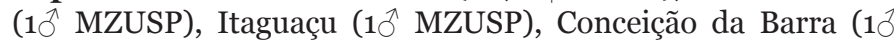
DZUP), Linhares (1 ${ }^{\lambda}$ MZUSP). São Paulo: São Paulo (1 ${ }^{\lambda}, 2$ ㅇ MZUSP), Caraguatatuba (3우 MZUSP), Barueri (1 $\overbrace{}^{\lambda}, 1+$ MZUSP), lha de Búzios (1우 MZUSP), Ribeirão Preto (1우 MZUSP). Rio de Janeiro: Itatiaia (12우 $4 \hat{\circ}$ MZUSP, 1 우 NHM), Ilha do Governador (1오 MZUSP), Itaguaí (1오 MZUSP), Rio de Janeiro (2 9 MZUSP), Santa Maria Madalena (Santo Antônio do ImbéEstrada) (3+ 1 1 $^{\Uparrow}$ MZUSP), Km 47 Estrada Rio de Janeiro-São

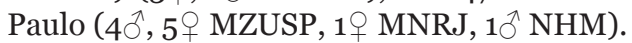

\section{Ommatius spinosus Scarbrough, 1993 (Figura 6)}

Scarbrough, 1993: 749, figs 121-131; 2007: 471; Vieira, Castro \& Bravo, 2005: 21 (chave); Papavero 2009: 52 (catálogo).

Comentários. O. spinosus (Figura 6) é registrada pela primeira vez para os estados de Rondônia e Mato Grosso. De acordo com a descrição original e com base no material-tipo examinado, $O$. spinosus possui uma ou duas cerdas pretas ou castanhas, longas e grossas e uma cerda amarelada, longa e fina no epândrio. Um espécime identificado do Pará difere do material-tipo por possuir três cerdas amareladas longas e finas.

Distribuição. EQUADOR; PERU; BOLÍVIA; BRASIL: Amazonas: Manaus. Pará: Belém, Fordlândia, Canindé-Rio Gurupí, Gorotire Xingu, Santarém. Maranhão: Igarapé GurupiUna (Aldeia Araçu). Rondônia: Vilhena (novo registro). Mato Grosso: $12^{\circ} 50^{\prime} \mathrm{S} 51^{\circ} 47^{\prime} \mathrm{W}$ (novo registro), $1^{\circ} 50^{\circ} \mathrm{S} 51^{\circ} 45^{\prime} \mathrm{W}$ (novo registro); Goiás: Corumbá [de Goiás], Jataí. Rio de Janeiro: Rio de Janeiro.

Material examinado. Parátipos: BK [Bert Klein coletor], (9.x.[19]85), Res.[erva] 1301, R.L.N.1, [BRASIL], [Amazonas], [ZF-03] [Km 23] / V. P. Daniel, Manaus / Paratype Ommatius spinosus Scarbrough (10 INPA). [BRASIL], Pará, Fordlândia, ii.1957, Pereira \& Machado / São Paulo Insect Collection / Paratype Ommatius spinosus Scarbrough (1우 MZUSP); Canindé, Rio Gurupí, Pará, BRASIL, vi.1963, B. Malkin col.[etor] / São Paulo Insect Collection / Paratype Ommatius spinosus Scarbrough (1 1 MZUSP). [BRASIL], Igarapé Gurupi-Una, Aldeia Araçu, MA[ranhão], 50 km E.[strada] de Canindé, ii.1966, Malkin col.[etor] / São Paulo Insect Collection / Paratype Ommatius

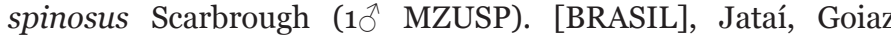
\{Goiás\}, i.1955, M. Carrera, A. Machado, F.S. Pereira, E. Dente, Milgar Loureiro col.[etor] / São Paulo Insect Collection / Paratype

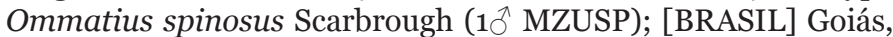
Corumbá \{[de Goiás]\}, F. Monjolinho, xi.l945, Barretto col.[etor] / São Paulo Insect Collection / Paratype Ommatius spinosus Scarbrough $(1 \hat{\jmath}, 1$ 우 MZUSP).

Material adicional. BR[ASIL], Amazonas, Manaus, ZF-O3, KM 23, Fazenda Esteio, Res.[erva] 1208, 10.xii.1986 / Malaise B.C. Klein Leg. / Ommatius spinosus Det. R. Vieira, 2007 (1 INPA). PA[rá], Gorotire Xingu, 18.iv.1983 / BRASIL, Pará, W. L. Overal / Ommatius spinosus Det. R. Vieira, 2007 (1へ MPEG); Mocombo, Belém, 1.x.1971 / Pará, BRASIL, T. Pimentel Col[etor] / Ommatius spinosus Det. R. Vieira, 2007 (1 ${ }^{\Uparrow}$ MZUSP). BRASIL, RO[ndônia], Vilhena 124655S 602218W, 25.iv.2006, J.A. Rafael \& F.F. Xavier $\mathrm{F}^{\mathrm{o}}$, arm. Malaise / Ommatius spinosus Det. R. Vieira, 2007 (1 $\overbrace{}^{\lambda}$ INPA). Gallery Forest / Brazil, \{BRASIL\}, Mato Grosso, $12^{\circ} 50^{\prime}$ S $51^{\circ} 47^{\prime} \mathrm{W}, 25 . i i i .1968$, O.W. Richards. / R.S. \& R.G.S. Expedition B.M. 1968-26o / Ommatius spinosus Det. R. Vieira, 2007 (1 $0^{\wedge}$ MZUSP); Gallery forest. / BRAZIL \{BRASIL\}, Mato Grosso, Base Camp. $12^{\circ} 50$ 'S 51 ${ }^{\circ} 45^{\prime} \mathrm{W} /$ 15.ii-8.iii.1968, B.E. Freeman.[Coletor] / Ommatius spinosus Scarbrough (1우 NHM).

\section{Ommatius uncatus Scarbrough, 1993}

Scarbrough, 1993: 750, figs 132-142; 2007: 472; Vieira, Castro \& Bravo, 2005: 20 (chave); Scarbrough 2007: 469 (chave); Papavero 2009: 52 (catálogo).

Comentários. Ommatius uncatus é registrada pela primeira vez para o Amazonas. De acordo com a descrição original de SCARBRough (1993) e com base no parátipo examinado, o fêmur mediano da fêmea possui duas a quatro cerdas marrons ânteroventrais e o fêmur posterior possui cinco a seis cerdas pretas ântero-ventrais e uma cerda basal amarelada ântero-ventral. No material adicional examinado do Amazonas houve as seguintes variações: o espécime fêmea do Tonantins possui somente uma cerda marrom ântero-ventral no fêmur mediano e oito cerdas ântero-ventrais (duas basais amareladas e as seis demais cerdas pretas) no fêmur posterior. No espécime do Amazonas, o fêmur posterior possui sete cerdas pretas ântero-ventrais.

Distribuição. PERU; COLÔMBIA; BRASIL: Amazonas: Bacaba (novo registro), São Gabriel da Cachoeira (novo registro), Tonantins (novo registro). Pará: Santarém.

Material examinado. Parátipo: [BRASIL], Fazenda Taperinha, Santarém, PA[rá], x-xi.1970, Exp. Perm. Amaz. / São Paulo Insect Collection / Paratype Ommatius uncatus Scarbrough (1우 MZUSP).

Material adicional. BRA[SIL], Amazonas, Pq. N. Unini, Bacaba, 014552 S, 620511 W, 14-19.vi.1996 / A. Henriques Leg. / Ommatius uncatus Det. R. Vieira, 2007 (19 INPA); BRASIL, AM[azonas], Tonantins, 025015S - 674630W, 16-20.ix.2005, arm.[adilha] luz, J. A. Rafael \& F.F. Xavier Fo / Ommatius uncatus Det. R. Vieira, 2007 (1 9 INPA); BRASIL, Est.[ado] do Amazonas, Mun.[icípio] São Gabriel da Cachoeira, Querari, $2^{\mathrm{O}}$ Pelotão de Fronteira ( $\left.2^{\mathrm{O}} \mathrm{PEF}\right) \mathrm{O}^{\circ} \mathrm{O} 5^{\prime} \mathrm{N}-69^{\circ} 51^{\prime} \mathrm{W} / \mathrm{0} 5$.iv27.v.1993, Motta C.S., Ferreira, R.L., Vidal, J. \& Matted, B. col. [etores] / Malaise / o065715 / Ommatius uncatus Det. R. Vieira, 2007 (1오 INPA).

\section{AGRADECIMENTOS}

Ao PPBio, CNPq e a FAPEAM EDITAL N. 022/2013 - FIXAM/ AM No Processo: 062.00745/2014. Aos curadores Dr. Augusto Henriques (INPA), Dr. Carlos Lamas (MZUSP), Dra. Márcia Couri (MNRJ), Dra. Luciane Marinoni (DZUP), Dr. Orlando Silveira (MPEG), Dr. Andreas Kohler (UNISC), Dr. James Boone $(\mathrm{CFMNH})$ e Dra. Erica McAlister (NHM) pelo empréstimo dos espécimes. Ao PPBIO Semi-árido por ter financiado as viagens para os municípios de Senhor do Bonfim e Morro do Chapéu na Bahia.

\section{REFERÊNCIAS}

Bigot, J.M.F., 1876. [Note: Descriptions of four new species of Asilidae]. Bulletin de la Societe entomologique de France, 6: 1xxxv-xxxvi.

Bromley, S.W., 1946. The robber flies of Brazil (Asilidae, Diptera). (Sociedade Brasileira de Entomologia) Livro de homenagem R. Ferreira d'Almeida, 8: 103-117.

Carrera, M. 1960. Asilidae (Diptera) da coleção Seabra. Arquivos de Zoologia do Estado de São Paulo, 11: 147-170.

Cezar, L.A. \& C.J.E., Lamas, 2010. Description of the larva and pupal case of Ommatius orenoquensis Bigot (Diptera, Asilidae, Ommatiinae). Revista Brasileira de Entomologia, 54: 28-31.

Cumming, J.M. \& D.M., Wood, 2009. Adult morphology and terminology, p. 9-50. In: Brown, B.V., Borkent, A., Cumming, J.M., Wood, D.M., Woodley, N.E. \& M.A. Zumbado (Eds.). Manual of Central American Diptera, Vol.1., National Research Council Research Press, Ottawa, Ontario, Canada, 950p. 
Curran, C.H., 1928. New species of Ommatius from America, with key. (Asilidae, Diptera). American Museum Novitates, 327: 1-6.

Dikow, T., 2009 Phylogeny of Asilidae inferred from morphological characteristics of imagines (Insecta: Diptera: Brachycera: Asiloidea). Bulletin of the American Museum of Natural History, 319: 1-175.

Hull, F.M., 1962. Robber Flies of the World: The Genera of the Family Asilidae. Bulletin of the United States National Museum, Part 2, 224: 431-906.

Kertész, C., 1909. Catalogus dipterorum hucusque descriptorum (IV). Oncodidae, Nemestrinidae, Mydaidae, Asilidae 4. Museum Nationale Hungaricum, 49-348.

Martin, C.H. \& N. Papavero, 1970. A catalogue of the Diptera of the Americas south of the United States, Family Asilidae. Boletim Museu de Zoologia, Universidade de São Paulo, 35b: 1-139.

Osten Sacken, C.R., 1891. Additions and corrections to the Catalogue of the described species of South American Asilidae by S. W. Williston. Berliner entomologie Zeitschrift, 36: 417428.

Papavero, N., 2009. Catalogue of Neotropical Diptera. Asilidae. Neotropical Diptera, 17: 1-179.

Scarbrough, A.G., 1990. Revision of the New World Ommatius Wiedemann (Diptera: Asilidae). I. The pumilus species group. Transactions of the American Entomological Society, 116: 65-102.

Scarbrough, A.G., 1985. Ommatius Wiedemann (Diptera: Asilidae) in the Lesser Antilles. Proceedings of the Entomological Society of Washington, 87: 641-655.

Scarbrough, A.G., 1993. Revision of the New World species of Ommatius Wiedemann (Diptera: Asilidae): the Neotropical costatus species group. Revista de Biologia Tropical, 41: 729753.

Scarbrough, A.G., 2000. Two additional species of robber flies of the Ommatius Wiedemann (Diptera: Asilidae) from the Bahamas and with replacement names for two other species. Proceedings of the Entomological Society of Washington, 102: 912-918.

Scarbrough, A.G., 2002. Synopsis of the Neotropical holosericeus complex of the genus Ommatius Wiedemann (Diptera): ampliatus and holosericeus species group. Transactions of the American Entomological Society, 128: 133-222.

Scarbrough, A.G., 2003. The Afrotropical Ommatius flavipennis species group (Diptera: Asilidae), with descriptions of six new species. Proceedings of the Entomological Society of Washington, 105: 611-629.

Scarbrough, A.G., 2005. Afroestricus, a new Afrotropical Ommatiinae (Diptera: Asilidae) genus with twenty species and two species groups. Zootaxa, 1041: 1-76.
Scarbrough, A.G., 2007. A new species of Ommatius Wiedemann from Brazil (Diptera: Asilidae) with notes on the Ommatius costatus species group. Transactions of the American Entomological Society, 133: 465-472.

Scarbrough, A.G., 2008. New Ommatius Wiedemann from the Americas with two new species groups, keys, and taxonomic notes (Diptera: Asilidae). Insecta Mundi, 32: 1-14.

Scarbrough, A.G. \& J. Constantino, 2005. The genus Ommatius Wiedemann, Dilatipennis species group (Diptera: Asilidae). Proceedings of the Entomological Society of Washington, 107: 789-807.

Scarbrough, A.G. \& C.G. Marascia, 2003. Revision of Ommatius Wiedemann (Diptera: Asilidae) IV. Pygommatius subgen. nov. with twenty-five Afrotropical species. Zootaxa, 228: 1-94.

Scarbrough, A.G. \& D.E. Perez-Gelabert, 2006. A review of the asilid (Diptera) fauna from Hispaniola with six genera new to the island, fifteen new species, and checklist. Zootaxa, 1381: 1-91.

Scarbrough A.G. \& G. Tomasovic, 2010. Ommatomyia, a new genus from Vietnam (Diptera: Asilidae: Ommatiinae). Zootaxa, 2366: 46-54.

Vieira, R., 2012. A new species of Leinendera Carrera, 1945 (Diptera, Asilidae, Asilinae) from Brazil. Biota Neotropica, 12: $1-7$.

Vieira, R., I. Castro \& F. Bravo, 2004. Two new species of Ommatius Wiedemann (Diptera: Asilidae) from Brazil. Zootaxa, 764: 1-7.

Vieira, R., I. Castro \& F. Bravo, 2005. A new species of Ommatius Wiedemann (Diptera: Asilidae) from Brazil. Zootaxa, 1017: 19-24.

Vieira, R., F. Bravo \& J.A. Rafael, 2010. Ommatius Wiedemann, 1821, normus species-group (Diptera, Asilidae): description of two new species and comments on Brazilian species. Zootaxa, 2344: 39-51.

Vieira, R., F. Bravo \& J.A. Rafael, 2011. O complexo holosericeus de Ommatius Wiedemann no Brasil: nova espécie e primeiro registro do grupo ampliatus para o País e novos registros para o grupo holosericeus (Diptera, Asilidae). Revista Brasileira de Entomologia, 55: 549-559.

\section{Recebido em: 29/o7/2014}

Aceito em: 16/11/2014

\section{Como citar este artigo:}

Vieira, R., 2015. O Grupo costatus de Ommatius Wiedemann (Diptera, Asilidae) no Brasil: Novos Registros e Distribuição de Ommatius costatus Rondani e Ommatius orenoquensis Bigot. EntomoBrasilis, 8 (1): 58-64.

Acessível em: $\underline{\text { doi:10.12741/ebrasilis.v8i1.461 }}$
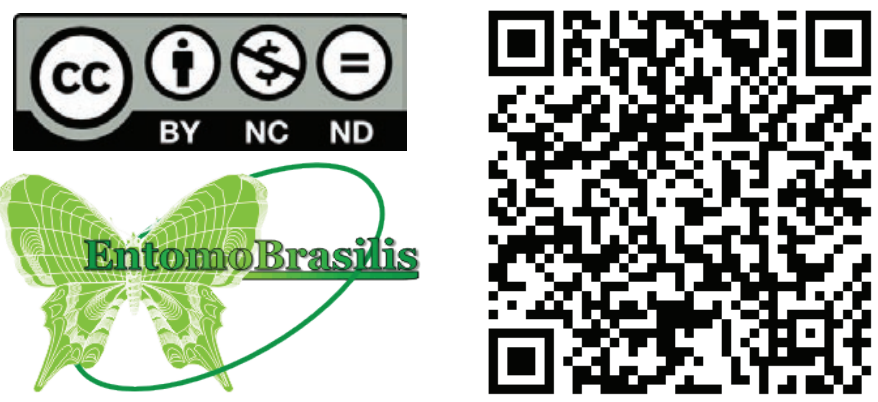